\title{
AUSCULTATORY BLOOD-PRESSURE DETERMINATIONS
}

\author{
A PRELIMTNARY REPORT \\ J. C. GITTINGS, M.D. \\ PHILADELPHIA
}

In October, 1905, Korotkow, of St. Petersburg, first advocated the determination of blood-pressure by auscultation. His method has been thoroughly studied, especially in Germany, and has been adopted by many observers as a routine procedure. Apparently it has been little, if at all, discussed in American or English literature, and in view of the importance of proving or disproving certain claims which have been made for it, it has seemed advisable to present this preliminary report of a series of observations which have been undertaken, in the hope that others may be induced to gather testimony.

The usual manometer and its equipment are employed in the auscultatory method, and the brachial artery is selected for auscultation at a point from two to four $\mathrm{cm}$. below the cuff. The blood-current is shut off in the usual manner and the first sound which is heard, as, with the fall of the mercury, the blood-current reaches the point selected for auscultation, corresponds to the maximum systolic pressure. Then follow variations in the sounds, which will be discussed at length, and finally, with the release of all pressure in the cuff, the disappearance of all sounds. The last phase, according to Ettinger, ${ }^{1}$ corresponds to the minimal or diastolic pressure.

Korotkow explains the first sound through the forcing apart of the vessel walls by the first stream of blood which reaches the artery below the cuff. He maintains that the lower part of the brachial artery, during the time that compression is exerted above it, is in a condition of complete relaxation and that the first blood-stream causes a sudden sharp stretching of the walls with the consequent production of sounds. Bozowski advanced the theory that the first and succeeding sounds originate in the heart and are transmitted by the blood-stream, but, as Krylow observes, this view is entirely negatived by the fact that all sounds cease with the release of compression.

Ehret ${ }^{2}$ believes that the suddenness of the distention, and not the amplitude of the blood-wave, determines the intensity of the sound. The

1. Ettinger: Wien. klin. Wchnschr., 1907, xx, 992.

2. Ehret: München. med. Wehnschr., 1909, Ivi, 959. 
fact that the sounds diminish a short distance below the cuff he ascribes to the less complete emptying of the artery, due, in turn, to peripheral arterial and venous pressure.

The theory that the sudden stretching of the relaxed vessel-wall produces the first sound is in accordance with the well-known physiological fact that independent sounds can originate in a blood-vessel only when the distention of that vessel at the moment of transmission of the bloodwave is at once sudden and of considerable intensity.

That the phenomena of the sounds are largely dependent on the size of the vessel as well as on its accessibility can be proved by the observation that while the cuff is applied to the forearm, the observer hears the sound exceptionally and only for a short time over the radial or ulnar artery. Finally it is evident that the resonating character of the cuff plays a most important rôle, since, with simple compression by an Esmarch bandage, the sounds are not, as a rule, audible.

Of greater interest than the determination of the maximal and minimal pressure is the interpretation of the intermediate sounds. The following sequence can be determined in the average case.

The cycle begins with the passage of the first waves of blood under the cuff. This produces a clear-cut sound resembling the tap of embryocardial rhythm and is known as the "first phase." Succeeding this a murmur becomes audible, more or less clearly defined and of varying duration. In the average case the murmur at first accompanies the sound but soon replaces it. The period of audible murmur is known as the "second phase." At the inception of the next or "third phase" the murmur disappears and is substituted by a clear sound. With the further fall of the mercury this clear sound becomes dull. At times this transformation is clear-cut and easily recognizable-again it occurs more gradually. Ettinger ${ }^{1}$ and Fischer, ${ }^{3}$ describe this transformation as the "fourth phase." The latter believes that it coincides with the diastolic pressure. Ettinger considers that it precedes true diastole and that the latter coincides with the disappearance of all sounds, which he calls the "fifth phase."

Krylow, in a previous conmunication, described only the first three phases. My own experience coincides with that of Ettinger and the differentiation into the five phases would seem to be possible in a large majority of cases.

In a discussion of these phases of the auscultatory phenomena it might be well to recall that, according to Landois and Corrigan, the rapidity of a blood-stream in a vessel is indirectly proportionate to the

3. Fischer: Ztschr. f. diätet. u. physik. Therap., October, 1908, p. 391. 
diameter of the lumen of that vessel. As the rapidity of the stream is the chief factor in the production of the sudden stretching of the vessel wall, which, in turn, produces the vibration which we hear as the arterial sound, this physiological fact becomes of much importance in the explanation of the auscultatory phenomena.

Krylow considers that the first sound produced is the loudest and ascribes this to the fact that, the lumen of the vessel under the cuff being at a minimum, the rapidity of the blood-stream is consequently maximal and thereby fulfills the conditions necessary for a sudden stretching of the walls of the vessel below the cuff.

Ettinger claims, however, that the greater volume of blood which passes under the cuff by the time the third stage is reached is capable of producing a more marked vibration of the vessel walls even though the rapidity of the current is less. With the appearance of the fourth phase the vessel walls are approaching a normal degree of expansion and vibration is consequently less marked. Added to this factor are the slowing of the blood-stream and the collapse of the cuff whereby it loses its resonating qualities. Ettinger found that in 90 per cent. of his 235 cases studied in Janowsky's clinic, the third phase was distinctly louder than the first. Fischer, from his study of 180 cases, is in practical accord with this opinion. The origin of the murmur during the second phase would seem to be due to the formation of whirls in the blood-stream, caused by the blood rushing from the obstruction at the cuff into the area of increased diameter below it. Friederich, in differentiating between murmurs and sounds, believed that the former depend on vibrations (Schwingungen) in the blood-stream, the latter on vibrations of the vessel walls.

In 1854 Heynsius stated that the essential factor in the production of a murmur was a narrowing of the lumen of the vessel and that rapidity of the current, to which Cheveau ascribed so much importance, alone was unable to produce a murmur. Friederich, believing with Cheveau that the rapidity of the blood-stream is a factor of great importance, considers that the viscosity of the blood, through its effect on the rate of flow, likewise influences the production of murmurs. The loud and prolonged murmur which Fischer heard over the brachial artery in certain anemic patients would seem to corroborate Friederich's view. This prolongation of the murmur (second phase) occurred at the expense of the third phase. At times, also, the first phase was lacking and the fourth phase was longer than normal. These phenomena occurred with a low diastolic pressure. 
In cases of high diastolic pressure, on the other hand, Fischer found that the first phase was always loud and clear and the second phase, when present, was short but clear. If the third phase were short, the fourth phase could not be heard.

In arteriosclerosis, with hardening and loss of elasticity of the vessel walls, the auscultatory phenomena, according to Krylow, are apt to be more pronounced, since the back-pressure at the cuff probably causes some dilatation of the vessel above it, while the lumen of the vessel is smaller than normal. Both of these factors cause an increased rapidity in the transmission of the blood-wave when pressure in the cuff is released, which in turn favors the vibration of the vessel-walls.

In high-grade thickening of the arterial walls, however, especially where calcification had occurred, Fischer found that the sounds were distinctly less loud than normal, the more so in the arm which showed the greater degree of hardening. According to Ettinger's experience the rapidity of the flow distinctly increases the auscultatory phenomena. This accords with the belief that these sounds are dependent on vibration in the vessel-walls.

Since Janowsky has demonstrated an increased pulse celerity in 60 per cent. of all cases of arteriosclerosis, Ettinger considers that we should be on guard against a possible hypertonia when we find in young individuals that the auscultatory sounds are very clear. This, however, needs confirmation. The factors which are to be considered, therefore, in the interpretation of the varying sounds, are:

1. The degree of narrowing of the vessel.

2. The elasticity of the vessel.

3. The size of the pulse-wave.

4. The rapidity of its transmission.

The first two in clinical practice, can only be gauged by the presence or absence of sclerotic thickening. The size of the pulse-wave can be inferred from the feel of the artery while the rapidity may be judged in part by the number of beats. Fischer believes that the character of the sounds heard over the vessels corresponds in general to the sounds which are heard over the heart. He considers that the third phase is the most important, and that from its characteristics we can surmise the degree of heart vigor (Gröss der Herzarbeit).

In two of Fischer's cases of weakness of the third phase, in which the efficiency of the heart was apparently unimpaired, the possibility of stenosis of the aortic orifice was considered. An autopsy, in one of these cases, proved the correctness of this assumption. In general he found that the intensity of the third phase corresponded to the height 
of the blood-pressure. In several exceptions to this rule-cases of nephritis with high pressure--beginning failure of the heart could be demonstrated.

The duration of the third phase Fischer also considers of great importance. In high pressure with loud sounds, the third phase was always prolonged. In one case of weak heart, the duration was short but became longer with improvement in the condition of the heart. In some cases of very high pressure, he heard a lighter, clear sound between the second and third phases.

Fischer also heard Traube's change of pulse in some cases, the sounds diminishing during inspiration, especially if it were deep, becoming more clear during expiration. I have observed this in several instances but am unable to indicate its significance.

From Krylow's experience, atypical auscultatory phenomena point to different degrees of insufficiency of the heart muscle: for example, in the absence of a murmur, to relative insufficiency; in the absence of sounds and the presence of a murmar, to well-marked weakness of the heart (vollständig Schwach). In these cases the amount of blood and the rapidity of the blood-stream are not sufficient to produce the sound.

As has been said, Ettinger finds that the second sound is louder than the first in 90 per cent. of his cases. In the remaining 10 per cent. the weakness of the second sound he considers to be of pathologic significance. His specific findings are as follows: In twenty-eight cases of cardiac insufficiency with dyspnea and edema, the third phase was absent nine times. Since this phase depends on a moderately large pulse-wave and moderate rapidity in its transmission, which in turn, depend upon a strong systole, the absence of these would point to heart-weakness.

In two other cases the second and fourth phases were absent and in four cases the second phase was lacking. These were all cases of high-grade heart-weakness. Ettinger believes that absence of the third phase alone denotes moderate weakness of the heart muscle while coincldent absence of the second and fourth phases indicates that the weakness has reached a high degree. In two of his cases, when there was improvement of the heart, there was a return of the third phase and in two others, with increase of the weakness, there followed a disappearance of the second and third phases. In a case of fatal pneumonia, with sudden cardiac failure, on the day of death there occurred a sudden disappearance of the second and third phases. He emphasizes these facts, namely, that in severe disturbances of compensation certain auscultatory phases ranish, while in severe anemias, where the blood flows more rapidly than normal, the auscultatory sounds, according to his experience, have been 
very clear. He suggests that in polycythemia with splenomegaly the reverse would probably be found.

Authorities are not in accord as to the exact determination of diastole by the auscultatory method. Some believe that the fourth phase, at the moment when the clear sound becomes dull, represents the true diastole. With Ettinger I am inclined to accept the fifth phase (disappearance of sounds) as the indication of diastole. On account of its length, the discussion of the subject may properly be left for a future article.

The practical application of the auscultatory test by the single operator is at least as easy as with any other method, provided certain conditions obtain. First and most important is the complete cooperation of the patient. Any morement of the arm, or body, even the slightest, may produce a sound which can easily be misinterpreted. Second, for the average operator, confusion is easily introduced by extraneous noises. Third, the cuff must fit perfectly. In an emaciated patient this perfection, especially with the Janeway cuff, is, at times, difficult to secure. Finally, in many cases of aortic regurgitation, the determination of diastolic pressure by auscultation is, obviously, impossible, owing to the presence of the well-known "pistol-shot" sound.

Against these objections can be set the greater accuracy afforded by auscultation and, especially, the more satisfactory determination of the diastolic pressure. Most of us, I am sure, have often been dissatisfied with our interpretation of mercurial oscillation, and although many are proficient in the determination from the pulse alone, this can hardly be considered as accurate as the auscultatory test.

In my examinations by the auscultatory method, I have followed, in the main, the recommendations of Ettinger, calculating diastole as the fifth phase. All the observations have been made on patients in the medical wards of the Presbyterian Hospital in the service of Dr. Joseph Sailer, to whom I wish to express my indebtedness.

The Stanton sphygmomanometer with the Janeway $14-\mathrm{cm}$. cuff was employed. At a point from 2 to $5 \mathrm{~cm}$. below the cuff, the brachial artery was auscultated with a double stethoscope. The mean of two or more observations was taken in almost every instance. An assistant took the manometer readings, in order to obviate the factor of personal equation. Sterzing employs a 6 -cm. cuff, claiming somewhat lower figures than those obtained with the broad cuff. The same author insists on the patient resting in bed for a half an hour before the tests, which should be made at the same hour each day in any comparative series of examinations.

4. Deutsch. med. Wehnsehr.. 190!, xxxv. 1874 . 
Fischer insists on the importance of allowing complete collapse of the cuff between observations in order to obviate congestion which might, in the presence of a weak heart, depress the systolic reading and cause the third phase to lose some of its force. The same author also rejects the readings in the first observations on nervous individuals, as the psychic disturbances must be discounted. Several improvements in recording my cases were made as the study advanced, so that some of the first results were not used, owing to a lack of sufficient data in reference to the variations in the different phases. The final scheme included the following:

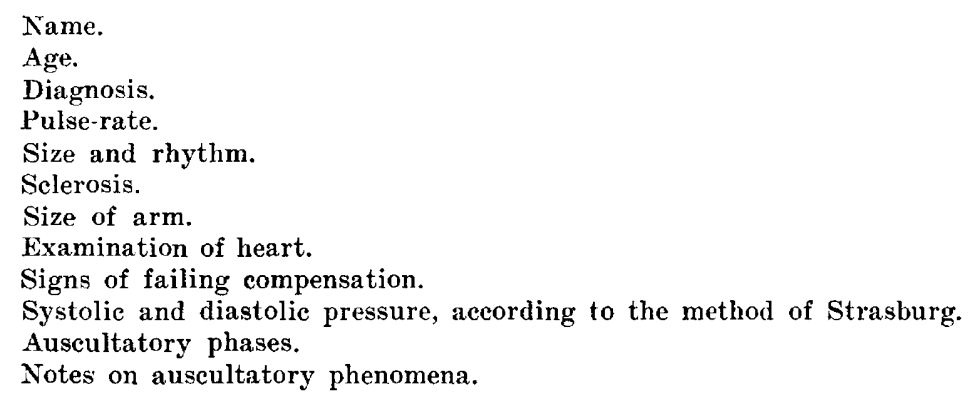

From the examination of sixty-three cases, which form the basis for this preliminary report, the following results may be tabulated:

In sixty-one cases, the auscultatory MX averaged $16.7 \mathrm{~mm}$. higher than that determined by palpation.

In two cases, the reverse occurred, the palpatory MX being higher than the auscultatory. In one case the difference was only $2 \mathrm{~mm}$., and in the other, $27 \mathrm{~mm}$. In the latter, however, the arm was excessively thin, and all the auscultatory phenomena were difficult to determine.

In forty-one cases, diastole, as determined by auscultation, averaged $15.5 \mathrm{~mm}$. lower than that determined by the visual method.

In five cases, diastole by auscultation averaged $7.7 \mathrm{~mm}$. higher than that by the visual method.

In two cases the results were equal.

In one case, diastole could not be determined by auscultation.

In eleven cases, diastole could not be determined by inspection.

The maximum pressure being higher by the auscultatory method and the minimum being lower, it follows that the pulse-pressure is greater in the auscultatory than in the visual method. Ettinger found an average difference of $13.2 \mathrm{~mm}$., while $\mathrm{my}$ results give a higher figure, $24 \mathrm{~mm}$. Finally, there remain to be considered the variations from the normal sounds heard during the cycle. 
In one case, there was absence of the second and fourth phases. This occurred in a patient of 74 years with myocarditis and nephritis, with enlarged heart, but weak sounds, especially at the base.

In one case there was absence of the third and fourth phases, in a patient with mitral and tricuspid regurgitation, exhibiting marked symptoms of ruptured compensation. In another case of mitral and trieuspid regurgitation, the second, third and fourth phases were absent. In a third case of mitral and tricuspid regurgitation, the third phase was absent. Arteriosclerosis was well marked in this patient. In another instance of abscence of the third phase, myocarditis and slight sclerosis were present. In another advanced case of valvular disease, aortic and mitral and tricuspid regurgitation, the second and third phases were absent.

In one case of myocarditis and marked sclerosis there was an absence of the fourth phase. [In one case of myocarditis with slight edema but little sclerosis there was the absence of the murmur.] In one case of general tuberculosis, two days before death, with profound asthenia, the second, third and fourth phases were almost indistinguishable. In two cases, the murmur persisted throughout the cycle. One was a case of carcinoma of the stomach; the other, of neurasthenia.

In all three of these cases, however, the patients being women, the arm was so thin as to prevent accurate fitting of the cuff. In one case of chronic asthma, during a severe exacerbation, the second, third and fourth phases were difficult to distinguish, but the excessive respiratory disturbance in the presence of a low pressure $(111 \mathrm{~mm}$.) was the evident canse.

In the following cases, no difficulty was experienced in determining all of the phases.

Arteriosclerosis with systolic aortic murmur.

Mitral regurgitation with marked arrhythmia and slight sclerosis with no signs of lost compensation.

Chronic endocarditis with acute rheumatism and pericarditis and good compensation.

Finally in one case of aortic regurgitation, the fifth phase could not be determined. In the remaining cases the auscultatory phases shower no change from normal and the cardiovascular symptoms were unimportant. On the basis of this small number of cases, definite conclusions are, of course, unwarranted.

The results of my observations agree, in the main, so closely with the published reports that it seems desirable to embody them, in their unfinished state, in a preliminary communication. That practical con- 
chusions of real value as to the efficiency of the cardiovascular system may be reached through the medium of Korotkow's method seems to be a justifiable hope.

3942 Chestnut Street.

ADDI'IONAT, HiBLIOGKAPHY

Moritz, F.: München. med. Wehnsehr., 1909, lvi, 321.

Schrumpf and Schabel: Miinchen. med. Wehnschr., 1909, lvi, 704.

Zabel: Berlin. klin. Wehnschr., 1909, xlvi, 1352.

Federn: Wien. klin. Welnnsehr., Feb. 11, 1909.

van Westenrijk, N.: Zt.schr. f. Klin. Med., 1908, 1ri. No. 26. 